\title{
Estudo da orelha contralateral na otite média crônica: "Efeito Orloff ${ }^{\circledR}$ " \\ Contralateral ear in chronic otitis media: "Orloff ${ }^{\circledR}$ Effect"
}

\author{
Ana Bárbara Scheibe ${ }^{1}$, Mariana M. Smith ${ }^{2}$, \\ Letícia P. Schmidt ${ }^{1}$, Viviane B. Schmidt ${ }^{3}$, \\ Cristina Dornelles ${ }^{4}$, Lúcia Helena S. K. Carvalhal's, \\ Lisiane Kruse', Sady S. da Costa
}

\section{Resumo}

I

Introdução: A otite média crônica (OMC) representa entidade de alta prevalência e distribuição mundial. Apesar da grande quantidade de estudos publicados a respeito, ainda não há, na literatura pertinente, consenso sobre a patogênese da OMC. Uma das hipóteses é a do continuum, que apresenta a otite média crônica como uma série de eventos contínuos, onde insultos iniciais desencadeiam uma cascata de alterações. Partindo então da idéia de continuum, pesquisamos a orelha contralateral (OCL) de indivíduos com diagnóstico de OMC, descrevendo as alterações encontradas. Forma de estudo: Clínico prospectivo randomizado. Material e Métodos: Foram selecionados 108 pacientes com diagnóstico de OMC nãocolesteatomatosa (OMC NC) ou colesteatomatosa (OMC C) acompanhados no ambulatório do Grupo de Pesquisa em Patologia da Orelha Média do Hospital de Clínicas de Porto Alegre. Nestes, foi realizada otoendoscopia com fibra óptica bilateral. A orelha mais estável foi considerada contralateral (OCL), sendo classificada como normal ou alterada (e as alterações descritas). Resultados: Dentre os pacientes avaliados, $59,2 \%$ portavam OMC NC e 40,8\% OMC C. $46,3 \%$ de todos os pacientes apresentaram alterações significativas na OCL. Dos pacientes com OMC C, 57\% apresentavam alteração na OCL, sendo que 39\% dos pacientes com OMC NC tinham OCL alterada. A alteração mais freqüentemente encontrada foi retração de MT, em ambos os grupos. Conclusões: Os dados encontrados sugerem que pacientes que apresentam OMC tem maior probabilidade de apresentarem patologia na OCL, O que corrobora a idéia que a OMC se trata de um evento constitucional e não isolado da orelha média.
Palavras-chave: otite média crônica, colesteatoma, orelha contralateral.

Key words: chronic otitis media, contralateral ear, cholesteatoma.

\begin{abstract}
Summary
I

ntroduction: Chronic otitis media (COM) is an entity of high prevalence and worldwide distribution. Despite being studied in depth, its pathogenesis is still a matter of discussion. One of the theories for its pathogenesis is the continuum. According to this theory, otitis media is described as a sequence of events, initiated by an insult that would lead to a cascade of events. Based on the conception of the Continuum, we studied the contralateral ear of patients with COM. Study design: Clinical prospective randomized. Material and Method: 108 patients were included in this study. All had been diagnosed as having COM (with or without cholesteatoma) and were being followed by the Research on Middle Ear Pathologies Group of the Hospital de Clínicas de Porto Alegre. Otoendoscopy with optic fiber was carried out on the affected ear just as in the contralateral ear, witch was classified as normal or abnormal. Results: $59,2 \%$ of all patients had COM with cholesteatoma and 40,8\% had COM without cholesteatoma. In $46,3 \%$ of patients the contralateral ear was found to have some abnormality. In the group of patients with COM with cholesteatoma, 57\% had abnormal contralateral ear, and in the other group the contralateral ear was found to be abnormal in 39\%. The abnormality more frequently found on both groups was retraction of the eardrum. Discussion: The results suggest that patients with COM have grater probability of having bilateral disease, witch corroborates the idea that COM is not only an isolated event on the middle ear, but a constitutional one, and, therefore, bilateral.
\end{abstract}

\footnotetext{
${ }^{1}$ Acadêmica da Faculdade de Medicina da Universidade Federal do Rio Grande do Sul ${ }^{2}$ Médica Residente do primeiro ano do Serviço de Otorrinolaringologia do Hospital de Clínicas de Porto Alegre. ${ }^{3}$ Acadêmica da Faculdade de Medicina da Universidade Luterana do Brasil.

${ }^{4}$ Bióloga do Grupo de Estudos de Patologia da Orelha Média do Hospital de Clínicas de Porto Alegre.

5 Mestranda do Departamento de Cirurgia da Faculdade de Medicina da Universidade Federal do Rio Grande do Sul. ${ }^{6}$ Médica Otorrinolaringologista.

Professor Adjunto do Departamento de Oftalmologia e Otorrinolaringologia da Universidade Federal do Rio Grande do Sul Grupo de Estudos de Patologia da Orelha Média - GEPOM

Serviço de Otorrinolaringologia - Hospital de Clínicas de Porto Alegre - HCPA

Endereço para correspondência: Mariana Magnus Smith - R. Artur Rocha, 825 - Porto Alegre - RS - CEP 90459-171 Tel: (0xx51) 3332.6002 E-mail: marimagnussmith@hotmail.com

Artigo recebido em 8 de novembro de 2001. Artigo aceito em 13 de dezembro de 2001.
} 


\section{INTRODUÇÃO}

A otite média crônica (OMC) é definida como a presença de alterações teciduais, de origem inflamatória, irreversíveis na orelha média ${ }^{1,2}$. Apresenta alta prevalência e distribuição mundial - características que somadas lhe conferem posição de "questão de saúde pública", mesmo nos dias de hoje ${ }^{3,4}$.

A OMC, sem dúvida, representa uma das grandes áreas de interesse dentro da otorrinolaringologia moderna, tendo em vista a ampla variedade de material de pesquisa publicado a respeito do assunto na literatura pertinente ${ }^{5,6,7,8}$. Talvez o ponto de maior interesse dentro do capítulo OMC seja exatamente a sua patogênese. Por quais caminhos chegamos às perfurações centrais e marginais? De que formas surgem e se expandem os colesteatomas? Mais intrigante ainda que tais questões: porque pacientes distintos, partindo de um mesmo insulto inicial, apresentam evoluções às vezes tão claramente diversas? Enfim, as questões acerca do assunto ainda são muitas e as respostas surgem como uma série de hipóteses propostas na literatura.

Em nosso grupo temos seguido o modelo de patogênese proposto pelo grupo de Minneapolis - a chamada teoria do continuum ${ }^{4,9,10}$. Segundo esta, a otite média parece existir ao longo de uma série contínua de eventos, de forma que, após um desencadeante inicial, uma otite serosa ou purulenta tornar-se-ia seromucóide, mucóide, e, finalmente, não havendo regressão do quadro, sobreviria a cronificação. Desta forma, a OMC não parece um evento isolado que surge em uma orelha especificamente doente. Parece, sim, ser o produto de uma série de eventos "constitucionais" do indivíduo.

Admitindo-se então, de acordo com a teoria do continuum, que as otites médias com efusão sejam um processo patológico inicial que possa evoluir até a cronificação, a questão que se impõe é: uma vez que a prevalência de efusão bilateral é alta ${ }^{11}$, também não deveria o ser a prevalência de OMC bilateral?

Sendo assim, acreditamos que a presença de OMC em uma orelha traz consigo maior probabilidade de encontrarmos alterações relacionadas na orelha contralateral (OCL). Partindo-se então deste raciocínio e de poucos dados já descritos na literatura ${ }^{12,13}$, nossa proposta no presente estudo é de apresentarmos as características da OCL de uma série de pacientes portadores de OMC.

\section{MATERIAL E MÉTODO}

Os pacientes selecionados para este estudo são acompanhados no ambulatório do Grupo de Estudos das Patologias da Orelha Média (GEPOM) do Hospital de Clínicas de Porto Alegre (HCPA). Neste ambulatório são atendidos pacientes com diagnóstico de OMC, colesteatomatosa (OMC C) ou não (OMC NC), sem tratamento cirúrgico prévio. Na primeira consulta são realizados anamnese detalhada e exame otológico composto por acumetria, otoscopia, otoendoscopia e otomicroscopia. Nos casos em que se faz necessária, é realizada limpeza cuidadosa e detalhada do conduto auditivo externo. A otoendoscopia com fibra óptica de $0^{\circ}$ e $4 \mathrm{~mm}$ de ambas orelhas é gravada de forma seqüencial, com identificação clara do paciente. A imagem gravada é então revisada e descrita em protocolos específicos de forma sistemática pelo orientador chefe do ambulatório, em reuniões semanais. Os pacientes atendidos neste ambulatório têm seus dados sobre sintomas - otorréia, vertigem, zumbido, perda auditiva, cefaléia e outros protocolados sistematicamente a cada consulta. Todos realizam audiometria tonal e vocal no Serviço de Audiologia do HCPA e estes dados são armazenados em protocolos específicos. Os exames complementares são solicitados caso a caso. A abordagem terapêutica proposta para tais pacientes é definida de acordo com a patologia encontrada.

Para realização deste trabalho foram analisados todos os pacientes em acompanhamento no ambulatório referido acima no período de agosto de 2000 a julho de 2001 , todos classificados como portadores de OMC NC (sem presença de colesteatoma ao exame otológico) ou OMC C (quando da presença de colesteatoma ao exame). A OCL foi definida como a orelha normal, a orelha assintomática (quando ambas orelhas eram alteradas) ou como a orelha menos sintomática (nos casos em que ambas orelhas eram sintomáticas).

As otoendoscopias das OCL foram revisadas, sendo tais orelhas classificadas como normais ou alteradas. As alterações foram classificadas como: efusão, retração, perfuração, colesteatoma ou com presença de seqüelas (presença de neotímpano ou timpanoesclerose). As retrações de membrana timpânica foram definidas como de pars tensa, de pars flacida ou mistas (quando havia retração de ambas porções da membrana). Estas foram classificadas como leves, moderadas ou severas, de acordo com os critérios expostos na Tabela 1. Cada paciente foi protocolado em formulários específicos quanto à OCL.

Todos os participantes do estudo autorizaram o armazenamento dos dados e a filmagem da otoendoscopia.

\section{RESULTADOS}

Dentre os 108 pacientes estudados, 64 (59,2\%) apresentavam OMC NC e $44(40,8 \%)$ apresentavam OMC C. Avaliando o grupo total de pacientes, $84(77,8 \%)$ destes apresentavam alguma das alterações descritas acima à otoendoscopia na OCL.

No grupo de pacientes com OMC NC, 16 (25\%) apresentavam membrana timpânica (MT) absolutamente normal. As alterações mais encontradas nos $75 \%$ restantes foram as retrações de MT, que estavam presentes em 28 pacientes com OMC NC, sendo 16 casos de retração isolada de pars tensa (11 leves, 4 moderadas e 1 severa), 5 de 
retração isolada de pars flacida ( 2 leves, 2 moderadas e 1 severa) e 7 casos de retração tanto de pars tensa quanto de pars flacida - retração mista - (2 leve/moderada e 5 moderada/severa). As demais alterações encontradas foram: perfuração em 9 pacientes (14\%), timpanoesclerose em $6(9,37 \%)$, neotímpano em $2(3,1 \%)$, efusão em 2 (3,1\%), e colesteatoma em 1 caso $(1,56 \%)$.

Já dentre pacientes portadores de OMC C, 8 (18,1\%) apresentavam OCL sem qualquer alteração à otoendoscopia. As alterações encontradas mais freqüentemente também foram do grupo de retrações da MT, com um total de 24 pacientes. As retrações de pars tensa foram descritas em 11 pacientes (5 leves, 3 moderadas e 3 severas), as de pars flacida em 10 pacientes ( 3 leves, 5 moderadas e 2 severas), enquanto as retrações mistas estavam presentes em 3 pacientes (todos grau moderada/ severa). A segunda anormalidade mais comum no grupo de doentes com OMC C foi a presença de colesteatoma na OCL: 15,9\% dos casos (7). As demais alterações foram:

Tabela 1. Classificação das retrações de membrana timpânica. MT: membrana timpânica.

\begin{tabular}{lll}
\hline Localização & \multicolumn{1}{c}{ Grau } & \multicolumn{1}{c}{ Definição } \\
\hline Pars tensa & Leve & Apenas retração \\
& Moderada & $\begin{array}{l}\text { Toque da MT na articulação } \\
\text { incudoestapediana } \\
\end{array}$ \\
& Severa & Toque da MT no promontório \\
\hline Pars flacida & Leve & Apenas retração \\
& Moderada & Toque da MT no martelo \\
& Severa & Presença de erosão óssea atical \\
\hline Mista & Leve/moderada & Critérios acima \\
& Moderada/severa & Critérios acima \\
\hline
\end{tabular}

Tabela 2. Descrição das otoendoscopias das orelhas contralaterais $(\mathrm{OCL})$ estudadas. $\mathrm{OMC} \mathrm{NC}$ : otite média crônica não colesteatomatosa; OMC C: otite média crônica colesteatomatosa. PT: pars tensa. PF: pars flacida. M: mista. L/M: leve/ moderada. M/S: moderada/severa.

\begin{tabular}{lccc}
\hline Otoendoscopia & OMC NC & OMC C & OMC NC + C \\
\hline Normal & 16 & 8 & 24 \\
Retração PT Leve & 11 & 5 & 16 \\
Retração PT Moderada & 4 & 3 & 7 \\
Retração PT Severa & 1 & 3 & 4 \\
Retração PF Leve & 2 & 3 & 5 \\
Retração PF Moderada & 2 & 5 & 7 \\
Retração PF Severa & 1 & 2 & 3 \\
Retração M L/M & 2 & 0 & 2 \\
Retração M M/S & 5 & 3 & 8 \\
Efusão & 2 & 1 & 3 \\
Perfuração & 9 & 2 & 11 \\
Colesteatoma & 1 & 7 & 8 \\
Timpanoesclerose & 6 & 1 & 7 \\
Neotímpano & 2 & 1 & 3 \\
\hline TOTAL & 64 & 44 & 108 \\
\hline
\end{tabular}

presença de líquido (1), perfuração (2), neotímpano (1) e timpanoesclerose (1). A Tabela 2 descreve as alterações encontradas, separadamente entre OMC NC e OMC C e em conjunto.

Quando excluímos as retrações leves, a presença de líquido na orelha média, timpanoesclerose ou neotímpano, procurando avaliar, assim, as orelhas com alterações mais significativas, encontramos um total de 46,3\%, 25 (57\%) das OCL dos portadores de OMC NC e 25 (39\%) das OCL de pacientes com OMC C (Gráfico 1). A diferença entre estes dois grupos não é estatisticamente significativa.

Ao avaliar de forma global o grupo das retrações, 21 pacientes apresentaram retrações leves, 16 retrações moderadas e 15 pacientes tinham retrações severas. Do total de pacientes, $48,1 \%$ apresentaram algum grau de retração da membrana timpânica.

\section{DISCUSSÃO}

As OMC têm sido classificadas em dois grandes grupos: otite média crônica colesteatomatosa (OMC C) e otite média crônica não-colesteatomatosa $(\mathrm{OMC} \mathrm{NC})^{4}$. A esses grupos soma-se a otite média crônica silenciosa, onde há alterações teciduais irreversíveis presentes na fenda auditiva sem, entretanto, existir solução de continuidade na membrana timpânica ${ }^{1,6,14}$. O termo OMC simples (muitas vezes utilizado para caracterização de OMC NC) não nos parece adequado, uma vez que a ausência de colesteatoma não confere, na maioria das vezes, à patologia crônica da orelha média qualquer grau de "simplicidade".

Apesar das classificações de OMC serem didaticamente simples, não há consenso sobre este tópico na literatura, o que demonstra a clara dificuldade dos autores em geral na tentativa de descrever de forma estática esta patologia tão claramente dinâmica. Segundo a teoria do continuum não temos otite média aguda, otite média serosa, otite média secretora, otite média crônica colesteatomatosa

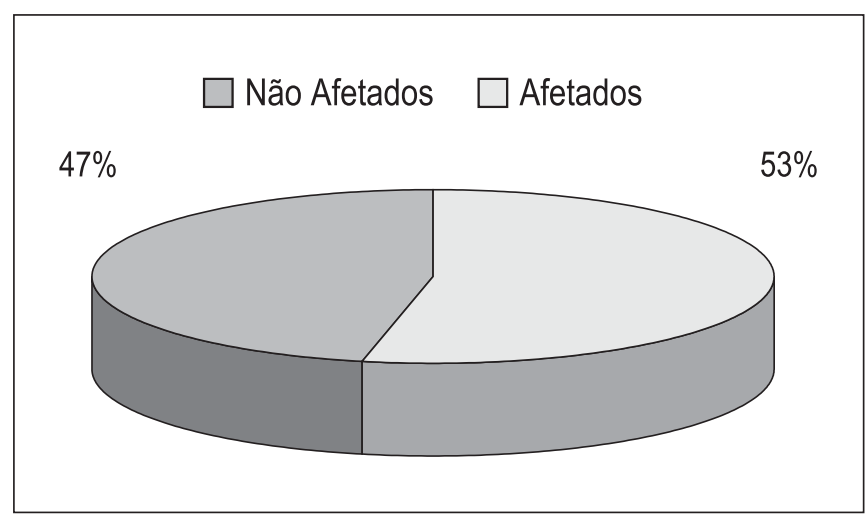

Gráfico 1. Distribuição de orelha contralateral (OCL) alteradas em pacientes com OMC, desconsiderando-se retrações leves, presença de efusão, neotímpano e timpanoesclerose. 
e não colesteatomatosa como entidades patológicas específicas com início, meio e fim. Temos, sim, uma mesma doença atravessando estágios evolutivos crescentes de eventos epiteliais e subepiteliais. Esta transição entre tais estágios far-se-á em um ou outro sentido dependendo basicamente do agente agressor, da orelha agredida e da atenção terapêutica que este paciente recebeu no curso de sua doença' ${ }^{5,10,15}$.

A concomitância de um processo patológico crônico da orelha média em um mesmo paciente já foi assunto de alguns trabalhos ${ }^{13,16}$. Entretanto, poucos estudos foram publicados descrevendo os achados de otoscopia da OCL de pacientes com OMC. Chalton \& Stears ${ }^{12}$ publicaram o primeiro estudo, em 1984, sobre tais achados. Os autores avaliaram as OCL de 73 pacientes submetidos à timpanomastoidectomia wall-down por colesteatoma adquirido e encontraram $53,4 \%$ de OCL alteradas, sendo a retração de pars tensa a alteração mais freqüente. Em 1996, Vartianen e colaboradores ${ }^{16}$ (1996) descreveram uma série de 493 OCL de pacientes com indicação de cirurgia otológica para OMC (OMC C e OMC NC). Estes autores encontraram 63\% das OCL com alguma anormalidade (definida como retração severa, perfuração ou colesteatoma), sendo a retração também o achado mais freqüente. Entretanto, nenhum destes trabalhos define de forma clara e objetiva os critérios utilizados para classificação das alterações encontradas, da mesma forma como não há definição de como é realizada a padronização da descrição.

A nossa série demonstrou alta prevalência de alterações na OCL de pacientes portadores de OMC. Quando excluímos as retrações leves e as seqüelas, analisando então apenas pacientes com alterações mais significativas na OCL, ainda encontramos prevalência de $46,3 \%$ de OCL anormais. Estes achados, então, corroboram a idéia de tendência à bilateralidade das patologias crônicas da orelha média.

Acreditamos na importância da valorização dos achados da OCL nos pacientes com OMC, seja ela OMC NC ou OMC C, por dois aspectos fundamentais: entendimento da patogênese da OMC e tratamento e aconselhamento de tais doentes.

Ao estudarmos as orelhas doentes aos pares, ou seja, ao sempre valorizarmos os achados da OCL, podemos de certa forma entender o processo patológico dinâmico que o paciente em questão nos evidencia. Não são raros os pacientes que apresentam de forma muito clara estágios diversos do processo patológico contínuo da OMC. São pacientes que, por exemplo, em um lado apresentam colesteatoma clássico atical e na OCL encontramos uma bolsa de retração atical, (ainda) sem colesteatoma. Ou que em um lado demonstram grande perfuração central da membrana timpânica e na OCL apresentam retração moderada difusa com nítida efusão presente na caixa.

A avaliação precisa e crítica de ambas orelhas de um determinado paciente, ao nosso ver, apresenta um papel fundamental na avaliação prognóstica do mesmo, uma vez que uma orelha com OMC estabelecida pode nos servir de guia para a provável evolução da OCL (ou mais estável). Se tivermos um paciente que de um lado apresenta um colesteatoma agressivo, com destruição ossicular importante e evolução desfavorável e na OCL apresenta bolsa de retração atical limpa, temos o dever de acompanhar de perto tal indivíduo, pois acreditamos que sua orelha "doente" está nos mostrando que esta bolsa de retração apresenta uma forte tendência à evolução também desfavorável.

Uma outra situação é àquela experimentada por pacientes submetidos à timpanotomia com tubo de ventilação bilateral. Não raramente um dos tubos é extruído mais precocemente, mantendo-se o outro in situ por períodos variáveis e, às vezes, prolongados. Apesar de não fazer parte da nossa rotina a remoção de um tubo de ventilação, algumas vezes consideramos esta hipótese estimulados pela ansiedade do paciente em voltar à plenitude da sua rotina. Acreditamos que para a decisão de remover ou não o tubo de ventilação deve ser avaliada a OCL. Se esta apresentar características normais, teremos então maior segurança para tal procedimento, uma vez que a OCL está nos mostrando que não tem tendência maior à progressão da doença.

Tais situações representam aquilo que chamamos informalmente em nosso ambulatório de "efeito Orloff ${ }^{\circledR}$ ", bebida bastante apreciada em nosso país cujo slogan é "eu sou você amanhã".

\section{CONCLUSÕES}

Os pacientes com OMC diagnosticada em uma orelha têm grande probabilidade de apresentarem alterações associadas na OCL, sendo a mais freqüente a retração de membrana timpânica.

Conseqüentemente, a OCL deve sempre ser minuciosamente avaliada em pacientes com OMC unilateral, com intuito de diagnosticar precocemente as alterações e, se necessário, de agir de forma terapêutica no momento mais oportuno.

\section{REFERÊNCIAS BIBLIOGRÁFICAS}

1. Costa SS, Paparella MM, Schachern PA et al. Histopatology of chronic otitis media with perforated and non-perforated tympanic membrane. Presented at Midwinter Meeting of the Association for Research in otolaryngology. Clearwater; 1989.

2. Meyerhoff WL, Kim CG, Paparella MM. Pathology of chronic otitis media. Ann Otol Rhinol Laryngol 1978;87(6):749-61.

3. Brown OE, Meyerhoff WL. Complications and sequelae of chronic suppurative otitis media. Ann Rhinol Otol Laryngol ; 1988: 97(131):38-40

4. Costa SS, Souza LCA, Piza MRT. The flexible endaural timpanoplasty. Pathology-guided, pathogenesis-oriented surgery for the middle ear. Otolaryngol Clin North Am 1999;32(3): 413-441. 
5. Junh SK, Paparella MM et al. Pathogenesis of otitis media. Ann Otol Rhinol Laryngol 1977;86(4):481-93.

6. Meyerhoff WL, Giebink GS, Shea DA. Silent otitis media: an animal study. Ann Otol Rhinol Laryngol 1984;93:136-9.

7. Paparella MM. Middle ear effusions. Definitions and terminology. Ann Otol Rhinol Laryngol 1976;85(25):8-11.

8. Sade J, Ar A. Middle ear and auditory tube. Middle ear clearance, as exchange and pressure regulation. Otolaryngol Head Neck Surg 1997;116:499-524

9. Giebink GG, Ripley MI et al. Clinical histopatological correlations in experimental otitis media: implications for silent otitis media in humans. Pediatr Res 1985;19(4):389-97.

10. Paparella MM, Hiraide F, Juhn SK, Kaneco J. Celular events involved in middle ear fluid production. Ann Rhinol Otol Laryngol 1970;79(4):766-79.
11. Casebrant M, Brostoff LM et al. Otitis media with effusion in preschool children. Laryngoscope 1985;95:428-36.

12. Chalton R, Stearns M. The incidence of bilateral chronic otitis media. J Laryngol Otol 1984;98:337-9.

13. Vartiainen E, Kansanen M, Vartiainen J. The contralateral ear in patients with chronic otitis media. Am J Otol 1996;17:190-2.

14. Costa SS. Contribuição ao estudo da otite média crônica. [Dissertação de Mestrado]. Faculdade de Medicina de Ribeirão Preto, USP; 1991.

15. Costa SS, Ruschel C, Cruz OL, Paparella MM. Otites Médias Aspectos Gerais. In: Cruz OL \& Costa SS. Otologia clínica e cirúrgica. 1999 b. Ed Revinter.

16. Vartiainen E, Kärjä J. Bilateral Otitis Media. Arch Otorhinolaryngol 1986; 243:190-3. 